\title{
Incidence and clinical characteristics of multiple myeloma with low M-protein levels and normal values of hemoglobin, creatinine, calcium, and serum free light chain ratio
}

\author{
Agoston Gyula Szabo (1)', Tobias Wirenfeldt Klausen², Niels Abildgaard ${ }^{3}$, Henrik Gregersen ${ }^{4}$, Trine Silkjær ${ }^{5}$, \\ Per Trøllund Pedersen ${ }^{6}$, Robert Schou Pedersen ${ }^{7}$, Carsten Helleberg ${ }^{2}$, Emil Hermansen $\mathbb{1}^{8}$, Brian Iversen Schnack ${ }^{1}$ and \\ Annette Juul Vangsted (10 ${ }^{9}$
}

\section{Dear Editor,}

The presence of an M-protein in the serum is a common incidental finding. It can be associated with infections, inflammatory conditions and autoimmune diseases, and the asymptomatic precursor conditions monoclonal gammopathy of undetermined significance (MGUS) and smoldering multiple myeloma (SMM). Only one percent of individuals with MGUS progress to a malignant plasma cell or lymphoproliferative disease per year ${ }^{1,2}$ and an important predictor of progression is the level of the Mprotein at the time of diagnosis, which is used in the riskstratification model by the Mayo Clinic ${ }^{2-4}$. Early diagnosis and treatment of multiple myeloma (MM) is important, as delays may result in life-threatening infections, painful bone fractures, or dialysis-dependent renal failure ${ }^{5-9}$. Moreover, there is increasing evidence supporting treatment of patients with SMM and this question is being explored in several ongoing clinical trials ${ }^{10}$. The serum free light chain (sFLC) assay, imaging of the skeleton, a bone marrow biopsy, and blood tests are part of the diagnostic work-up for MM, but if clinical evaluation and blood tests suggest low risk MGUS, a baseline bone marrow examination and skeletal radiography are not recommended by the International Myeloma Working Group $^{3,11}$.

\footnotetext{
Correspondence: Agoston Gyula Szabo (agoston.gyula.szabo@rsyd.dk) or Tobias Wirenfeldt Klausen (towikl01@gmail.com)

${ }^{1}$ Department of Hematology Vejle Hospital, Vejle, Denmark

2Department of Hematology Herlev University Hospital, Herlev, Denmark

Full list of author information is available at the end of the article
}

In a recently published retrospective review from the Mayo Clinic, Sidiqi et al. reported that 29 (1.3\%) of 2225 MM patients had laboratory values within reference range for calcium, creatinine, hemoglobin, a sFLC ratio $<100$, and absence of lytic lesions assessed by conventional skeletal survey. These patients would have been misclassified as SMM or MGUS without bone marrow biopsy or advanced imaging ${ }^{12}$.

The aim of our study was to describe the incidence of MM and SMM with an MGUS-like profile (defined as $\mathrm{MM}$ with IgG M-protein $\leq 1.5 \mathrm{~g} / \mathrm{dl}$ or an IgA M-protein $\leq 1.0 \mathrm{~g} / \mathrm{dl}$ and with normal levels of ionized calcium, creatinine, and hemoglobin) since 2005 in Denmark. Furthermore, we wanted to characterize the patients with MGUS-like MM who also had a normal sFLC ratio at diagnosis. Finally, we wanted to understand the reasons for referral for diagnostic work-up for these MM patients with no apparent signs of organ damage.

We used the population-based Danish Multiple Myeloma Registry (DMMR), which includes clinical data for all patients with MM and SMM diagnosed since $2005^{13-15}$. Measurements of sFLC have been recorded in the DMMR since 2012. A sFLC ratio of $0.26-1.65$ at diagnosis was defined as normal. Patients with LDH levels above normal were standardized according to age. In patients with age $>70$ years the upper limit was $\geq 255 \mathrm{UL}$, and in patients with age $\leq 70$ the upper limit was $\geq 205$ UL. Hypogammaglobulinemia was defined qualitatively as one or more of uninvolved immunoglobulins below normal levels $\quad(\operatorname{IgG}<6.1 \mathrm{~g} / \mathrm{L}, \quad \operatorname{IgA}<0.70 \mathrm{~g} / \mathrm{L}, \quad \operatorname{IgM}<0.39 \mathrm{~g} / \mathrm{L})$. High-risk cytogenetics were defined as the presence of 
A

Patients with newly diagnosed MM or SMM in the DMMR 2005-2018 (5116)

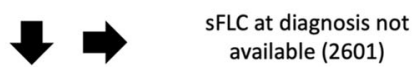

SFLC at diagnosis available (2515)

$7 \Rightarrow$ sFLC ratio abnormal (1730)

Normal sFLC ratio (785)

Hemoglobin $<8.3 \mathrm{mmol} / \mathrm{L}$ (men) or $<7.3 \mathrm{mmol} / \mathrm{L}$ (women)

Creatinine $>105 \mu \mathrm{mol} / \mathrm{L}$ (men) or $>90 \mu \mathrm{mol} / \mathrm{L}$ (women)

lonized $\mathrm{Ca}>1.32 \mathrm{mmol} / \mathrm{L}$

Spinal cord compression syndrome

Extramedullary myeloma

Hyperviscosity syndrome

Dialysis-dependent renal failure

Non-secretory MM

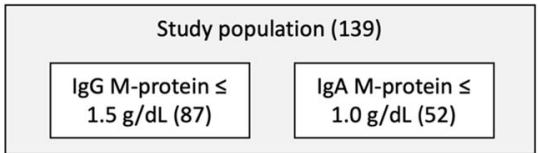

(646)

B

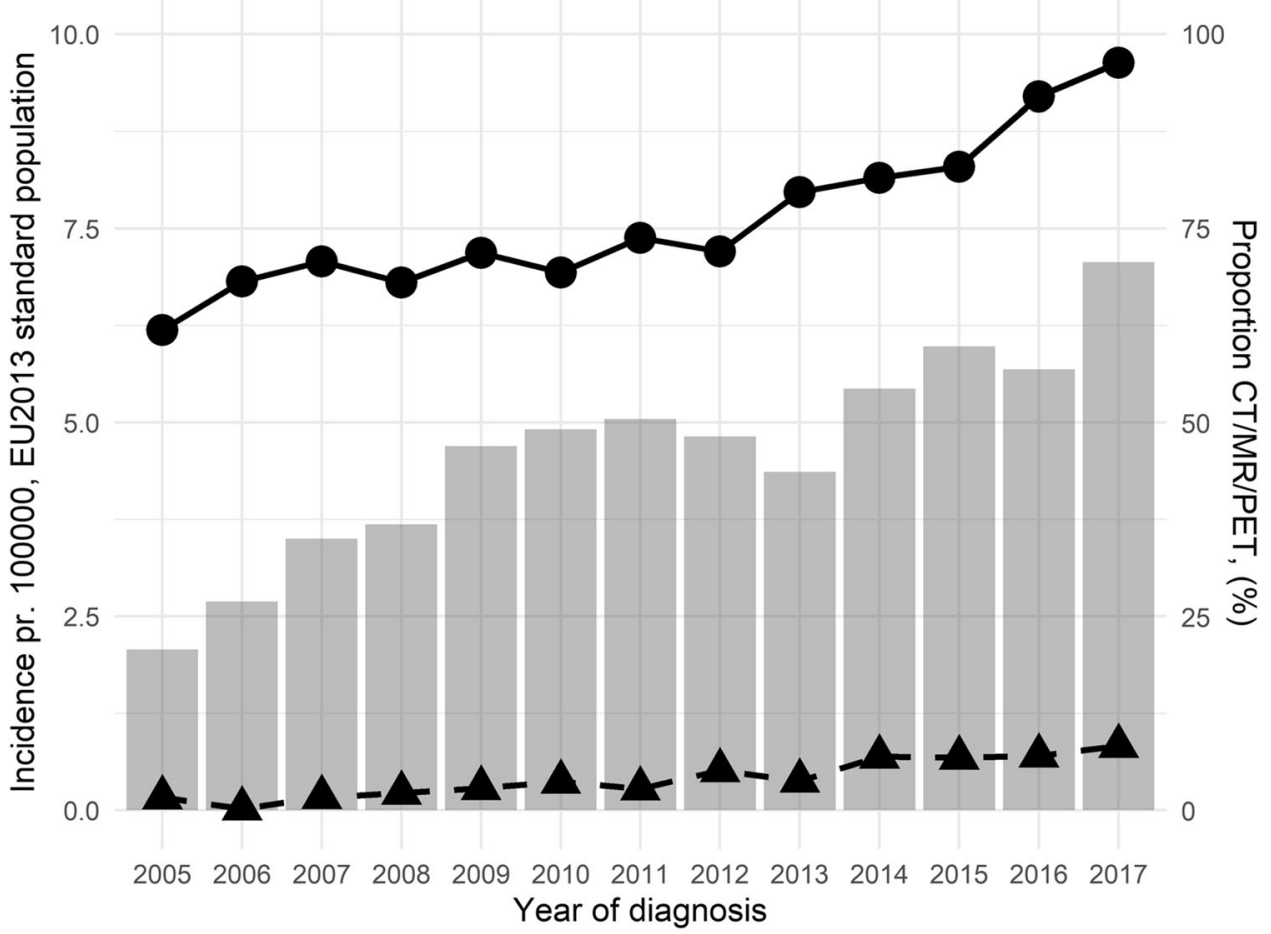

Fig. 1 Flow-chart for study inclusion and the incidence of multiple myeloma in Denmark. A Flow-chart for study inclusion: Among 5116 patients diagnosed with MM or SMM and registered in the DMMR, a diagnostic SFLC result was available in 2515 patients and 785 had a normal sFLC ratio. Of these patients, 139 had an MGUS-like phenotype IgG M-protein $\leq 1.5 \mathrm{~g} / \mathrm{dl}$ or an IgA M-protein $\leq 1.0 \mathrm{~g} / \mathrm{dl}$ and with normal levels of normal hemoglobin, creatinine, ionized calcium and no amyloidosis, medullary compression syndrome, extramedullary myeloma, peripheral neuropathy or dialysis-dependent renal failure at presentation. MM multiple myeloma, SMM smoldering myeloma, sFLC serum free light chain. B Incidence of multiple myeloma in Denmark: The columns with values displayed on the left vertical axis show the incidence of multiple myeloma pr. 100.000 in Denmark, age adjusted to the EU 2013 standard population. Filled circles present the age-adjusted incidence for all MM patients and the filled triangles present the age-adjusted incidence of myeloma patients with an IgG M-protein $\leq 1.5 \mathrm{~g} / \mathrm{dL}$ and $\operatorname{lgA} \mathrm{M}$-protein $\leq 1.0 \mathrm{~g} / \mathrm{dL}$ and normal blood levels of ionized calcium, creatinine, and hemoglobin. The gray bars with values displayed on the right vertical axis show the percentage of patients assessed with sensitive imaging techniques like CT, MRI, and PET (CT/MR/PET) as part of the diagnostic work-up. The years of diagnosis in the calendar period 2005-2017 are shown on the horizontal axis. Patients diagnosed in 2018 were not fully registered at data cut-off. 
del17p, $t(4 ; 14)$, or $t(14 ; 16)$ with a cut-off of $10 \%$. Patients with AL amyloidosis, medullary compression syndrome, peripheral neuropathy, or dialysis-dependent renal failure at presentation were excluded. The incidence of MGUSlike MM patients was age-adjusted to the EU 2013 population. Causes of diagnostic work-up were reviewed by audit of medical records. Data were entered in a Research Electronic Data Capture (REDcap) database and merged with the baseline characteristics from DMMR.

At the time of data cut-off for this study (3rd April 2019), the DMMR included 5116 newly diagnosed myeloma patients (Fig. 1A). We found a significant increase in the age-adjusted incidence of MM between 2005 and 2017 ( $p<0.001$; Fig. 1B). This increase was also present in MGUS-like MM with an IgG M-protein $\leq 1.5 \mathrm{~g} / \mathrm{dl}$ or an IgA M-protein $\leq 1.0 \mathrm{~g} / \mathrm{dl}$ with normal hemoglobin, creatinine, and ionized calcium at diagnosis $(p<0.0001$; Fig. 1B). The increase in the incidence of MM correlated significantly with a wider use of sensitive imaging techniques in the diagnostic work-up, such as CT, PET-CT, or MRI $(r=0.86 ; p=0.0002$; Supplementary Fig. 1$)$. The diagnostic sFLC ratio had a stronger correlation to the bone marrow clonal plasma cell percentage compared to the diagnostic M-protein concentrations (Supplementary Figs. 2 and 3).

We identified 139 patients (5.5\% of patients in the DMMR with available sFLC results), with MGUS-like MM. The clinical characteristics of these patients are presented in Supplementary Table 1. Osteolytic lesions were found in $23.9 \%$. No patients had higher than $60 \%$ bone marrow clonal plasma cell infiltration. The results of FISH analysis were available in $51.1 \%$ of the patients. High-risk cytogenetics were found in $12.7 \%$ of patients. Hypogammaglobulinemia, elevated Beta-2-microglobulin and elevated $\mathrm{LDH}$ were found in $52.9 \%, 34.5 \%$, and $15.4 \%$ of patients, respectively.

Causes of diagnostic work-up were available in all patients but one (Supplementary Table 2). Eighty (58.0\%) patients were referred with symptoms, most frequently bone pain and fatigue. Ninety-one (65.9\%) patients were referred with abnormal blood tests, most frequently the presence of a serum M-protein. Fourteen (10.1\%) patients were referred with an abnormal skeletal imaging result. Eight $(5.8 \%)$ patients were referred because of a biopsy finding. The number of patients without bone pain or abnormal skeletal imaging results at referral was 92 (67.2\%), of these 38 (41.8\%) had bone lesions. Fifty-eight patients $(42.0 \%)$ did not have any symptoms at referral; of these 27 (29.5\%) had bone lesions.

Data from the DMMR show an increase in the ageadjusted incidence of MM over the years that correlates with the wider use of sensitive imaging techniques. The incidence of MGUS-like MM has also increased from $0.12 / 100.000$ in the calendar period $2005-2007$ to 0.74 /
100.000 in the calendar period 2015-2017. In daily practice, the decision whether a bone marrow biopsy and sensitive imaging techniques should be performed in such patients is difficult, and there is an inherent risk of misdiagnosing MM as MGUS. In our population-based registry, $5.5 \%$ of $\mathrm{MM}$ patients had MGUS-like MM. Although bone marrow sampling did not have therapeutic implications in our population, our findings indicate that even in patients referred with low M-protein concentrations, normal sFLC ratio and normal levels of hemoglobin, creatinine and ionized calcium, careful evaluation of symptoms and sensitive imaging techniques should be considered.

\section{Acknowledgements}

Data management for this study was provided by Open Patient Exploratory Network, University of Southern Denmark. This study received funds from Tømrermester Jørgen Holm og Hustru Elisa F. Hansens Mindelegat. The authors received no further financial support for the research, authorship, or publication of this article.

\section{Author details}

${ }^{1}$ Department of Hematology Vejle Hospital, Vejle, Denmark. ${ }^{2}$ Department of Hematology Herlev University Hospital, Herlev, Denmark. ${ }^{3}$ Department of Hematology Odense University Hospital, Odense, Denmark. ${ }^{4}$ Department of Hematology Aalborg University Hospital, Aalborg, Denmark. ${ }^{5}$ Department of Hematology Aarhus University Hospital, Aarhus, Denmark. ${ }^{6}$ Department of Hematology Esbjerg Hospital, Esbjerg, Denmark. 'Department of Hematology Regionshospitalet Holstebro, Holstebro, Denmark. ${ }^{8}$ Department of Hematology Zealand University Hospital, Roskilde, Denmark. ${ }^{9}$ Department of Hematology Rigshospitalet, Copenhagen, Denmark

\section{Author contributions}

A.G.S. established the Redcap database, collected clinical data, interpreted the results, wrote the manuscript together with A.J.V. and approved the final version, T.W.K. performed all statistical analysis, interpreted the results, revised the manuscript and approved the final version, NA/HG/TS/PTP/SCP/CH/EH/BIS collected clinical data, interpreted the results, revised the manuscript and approved the final version, A.J.V. conceived and designed the work, collected clinical data, interpreted the results, wrote the manuscript and approved the final version.

\section{Conflict of interest}

The authors declare no competing interests.

\section{Publisher's note}

Springer Nature remains neutral with regard to jurisdictional claims in published maps and institutional affiliations.

Supplementary information The online version contains supplementary material available at https://doi.org/10.1038/s41408-021-00460-0.

Received: 10 December 2020 Revised: 4 March 2021 Accepted: 17 March 2021

Published online: 07 April 2021

\section{References}

1. Wadhera, R. K. \& Rajkumar, S. V. Prevalence of monoclonal gammopathy of undetermined significance: a systematic review. Mayo Clin. Proc. 85, 933-942 (2010).

2. Kyle, R. A. et al. Long-term follow-up of monoclonal gammopathy of undetermined significance. N. Engl. J. Med. 378, 241-249 (2018).

3. Kyle, R. A. et al. Monoclonal gammopathy of undetermined significance (MGUS) and smoldering (asymptomatic) multiple myeloma: IMWG consensus 
perspectives risk factors for progression and guidelines for monitoring and management. Leukemia 24, 1121-1127 (2010).

4. Rajkumar, S. V. et al. Serum free light chain ratio is an independent risk factor for progression in monoclonal gammopathy of undetermined significance. Blood 106, 812-817 (2005).

5. Sørrig, R., Klausen, T. W., Salomo, M., Vangsted, A. \& Gimsing, P. Risk factors for blood stream infections in multiple myeloma: a population-based study of 1154 patients in Denmark. Eur. J. Haematol. 101, 21-27 (2018).

6. Holmström, M. O. et al. Causes of early death in multiple myeloma patients who are ineligible for high-dose therapy with hematopoietic stem cell support: a study based on the nationwide Danish Myeloma Database. Am. J. Hematol. 90, E73-E74 (2015).

7. Hameed, A., Brady, J. J., Dowling, P., Clynes, M. \& O'Gorman, P. Bone disease in multiple myeloma: pathophysiology and management. Cancer Growth Metastasis 7, 33-42 (2014)

8. Terpos, E., Ntanasis-Stathopoulos, I. \& Dimopoulos, M. A. Myeloma bone disease: from biology findings to treatment approaches. Blood 133, 1534-1539 (2019).

9. Evison, F. et al. A population-based study of the impact of dialysis on mortality in multiple myeloma. Br. J. Haematol. 180, 588-591 (2018).
10. Mateos, M.-V. et al. Lenalidomide plus dexamethasone versus observation in patients with high-risk smouldering multiple myeloma (QuiRedex): long-term follow-up of a randomised, controlled, phase 3 trial. Lancet Oncol. 17 1127-1136 (2016)

11. Rajkumar, S. V. Updated diagnostic criteria and staging system for multiple myeloma. Am. Soc. Clin. Oncol. Educ. Book 35, e418-e423 (2016).

12. Sidiqi, M. H. et al. The role of bone marrow biopsy in patients with plasma cell disorders: should all patients with a monoclonal protein be biopsied? Blood Cancer J 10, 52 (2020).

13. Gimsing, P. et al. The Danish National Multiple Myeloma Registry. Clin. Epidemiol. 8, 583-587 (2016).

14. Abildgaard, $\mathrm{N}$. et al. Continued improvement in overall survival in elderly multiple myeloma patients after 2008; a population based study from the Danish Multiple Myeloma Registry. Clin. Lymphoma Myeloma Leuk. 15, e189 (2015).

15. Gregersen, $\mathbf{H}$. et al. The impact of comorbidity on mortality in multiple myeloma: a Danish nationwide population-based study. Cancer Med. 6, 1807-1816 (2017) 